\title{
THE DUAL OF THE SPACE OF HOLOMORPHIC FUNCTIONS ON LOCALLY CLOSED CONVEX SETS
}

\author{
José Bonet, Reinhold Meise and Sergej N. Melikhov
}

$\begin{array}{ll}\text { Abstract } & \text { Let } H(Q) \text { be the space of all the functions which are holomor- } \\ \text { phic on an open neighbourhood of a convex locally closed sub- } & \text { set } Q \text { of } \mathbb{C}^{N} \text {, endowed with its natural projective topology. We } \\ \text { characterize when the topology of the weighted inductive limit of } \\ \text { Fréchet spaces which is obtained as the Laplace transform of the } \\ \text { dual } H(Q)^{\prime} \text { of } H(Q) \text { can be described by weighted sup-seminorms. } \\ \text { The behaviour of the corresponding inductive limit of spaces of } \\ \text { continuous functions is also investigated. }\end{array}$

\section{Introduction}

Martineau investigated in [15] the spaces $H(Q)$ of analytic functions on a convex nonpluripolar set $Q$ in $\mathbb{C}^{N}$ in the case that $Q$ admits a countable fundamental system of compact sets. This setup covers nonpluripolar compact convex sets and convex open sets in $\mathbb{C}^{N}$ and convex open sets in $\mathbb{R}^{N}$. In the latter case $H(Q)$ coincides with the space of all real analytic functions on $Q$. The strong dual $H(Q)_{b}^{\prime}$ of each of these spaces can be canonically identified, via the Laplace transform, with a weighted (LF)-space $V H\left(\mathbb{C}^{N}\right)$ of entire functions on $\mathbb{C}^{N}$, i.e. $H(Q)_{b}^{\prime}$ is isomorphic to a Hausdorff countable inductive limit of Fréchet spaces of entire functions defined by weighted sup-seminorms. See the details below. The description of the topology of such weighted inductive limits of spaces of holomorphic functions has been investigated thoroughly in recent years since the work of Ehrenpreis [10] on analytically uniform spaces. Similar questions on the projective description were later investigated by various authors (see [1] ).

2000 Mathematics Subject Classification. Primary: 46E10; Secondary: 46A13.

Acknowledgement: The research of J. Bonet and R. Meise was partially supported by MEC and FEDER Project MTM2004-02262 and AVCIT Grupo 03/050. S. N. Melikhov thanks the support by DAAD and by Russian Foundation of Basic Research (project no. 02-01-00372). 
Many results about the projective description of weighted $(L F)$-spaces of entire functions were obtained in $[\mathbf{4}],[\mathbf{5}],[\mathbf{6}],[\mathbf{7}]$. In particular, we derived in $[\mathbf{6}]$ necessary as well as sufficient conditions for the projective description to hold algebraically or topologically for the space of Laplace transforms of $H(Q)_{b}^{\prime}$ where $Q$ is a bounded locally closed convex subset of $\mathbb{C}^{N}$. In the present paper we continue these investigations for such sets $Q$ which are not necessarily bounded. As in [6] we have algebraic and topological projective descriptions if $Q$ is strictly convex at the relative boundary of $Q \cap \partial_{r} Q$ (see Definition 2.4 and Theorem 3.1). In Theorem 3.11 we show that this condition is necessary for the topological projective description if we assume in addition that $Q$ admits a neighbourhood basis of domains of holomorphy. To obtain this result, we use a method different from that of our earlier work. The new idea is to apply the approach that was used in [5]. We use a result of Mal'tsev [14] which implies that if a convex, locally closed set $Q$ has a neighbourhood basis of domains of holomorphy and there is a supporting hyperplane to the closure of $Q$ with non-compact intersection with $Q$, then there is a differential operator $P(D)$ on $H(Q)$ which is not surjective.

Bierstedt and Bonet [2] extended the projective description techniques from (LB) to (LF) spaces of continuous functions using Vogt's approach [21] to Palamodov and Retakh theory of (LF)-spaces. The problem of topological projective description for the spaces $V C\left(\mathbb{C}^{N}\right)$ of continuous functions corresponding to the Laplace transform of the space $H(Q)_{b}^{\prime}$ has a positive answer by a result of Bierstedt, Meise, and Summers $[\mathbf{3}]$. The algebraic coincidence of the $(\mathrm{LF})$-space $V C\left(\mathbb{C}^{N}\right)$ and its projective hull is characterized in Theorem 4.5 by the condition that $Q$ is strictly convex at the relative boundary of $Q \cap \partial_{r} Q$.

\section{Notation and Preliminaries}

2.1. Definition. A subset $Q$ of $\mathbb{C}^{N}$ is called locally closed if for each $z \in Q$ there is a closed neighbourhood $U$ of $z$ in $\mathbb{C}^{N}$ such that $Q \cap U$ is closed. Every open subset and every closed subset of $\mathbb{C}^{N}$ is locally closed.

For a convex set $Q \subset \mathbb{C}^{N}$ the symbols $\operatorname{int}_{r} Q$ and $\partial_{r} Q$ denote the relative interior and the relative boundary of $Q$ with respect to the affine hull of $Q$. For example, if $0 \in Q$, the affine hull of $Q$ is the real linear span of $Q$. We write $\omega:=Q \cap \partial_{r} Q$. By $\partial_{r} \omega$ we denote the relative boundary of $\omega$ with respect to $\partial_{r} Q$.

2.2. Remark. By [6, Lemma 1] and [18, Lemma 1.2], the following assertions are equivalent for a convex subset $Q$ of $\mathbb{C}^{N}$ :

(i) $Q$ is locally closed. 
(ii) $Q$ admits a countable fundamental system of compact subsets.

(iii) $Q$ is the union of the relative interior $\operatorname{int}_{r} Q$ of $Q$ and a subset $\omega$ of $\partial_{r} Q$ which is open in $\partial_{r} Q$.

2.3. General Assumption. Throughout this article $Q$ denotes a locally closed convex set and $\left(Q_{n}\right)_{n \in \mathbb{N}}$ a fixed increasing fundamental sequence of compact convex sets in $Q$.

2.4. Definition ([18, Definition 1.3]). A locally closed convex set $Q$ is called $(\mathbb{C}$-) strictly convex at the relative boundary of $\omega$ if the intersection of $Q$ with each supporting (complex) hyperplane to the closure $\bar{Q}$ of $Q$ is compact.

If the interior of $Q$ is empty, the set $Q$ is strictly convex at the relative boundary of $\omega$ if and only if $Q$ is compact. If the interior of $Q$ is not empty, $Q$ is $(\mathbb{C}$-)strictly convex at the relative boundary of $\omega$ if and only if each line segment (of which the $\mathbb{C}$-linear affine hull belongs to some supporting hyperplane of $\bar{Q}$ ) of $\omega=Q \cap \partial_{r} Q$ is relatively compact in $\omega$. By [6, Proposition 2], a locally closed convex set $Q$ is strictly convex at the relative boundary of $\omega$ if and only if $Q$ has a neighbourhood basis of convex domains. For example $Q$ is strictly convex at the relative boundary of $\omega$ if $Q$ is open or compact. Let $Q \subset \mathbb{C}^{N}$ be a convex locally closed set such that $\bar{Q}:=\left\{x \in \mathbb{C}^{N}=\right.$ $\left.\mathbb{R}^{2 N} \mid x_{1} \geq f\left(x_{2}, \ldots, x_{2 N}\right),\left(x_{2}, \ldots, x_{2 N}\right) \in \mathbb{R}^{2 N-1}\right\}$ for a convex function $f: \mathbb{R}^{2 N-1} \rightarrow \mathbb{R}$. If the function $f$ is strictly convex in the sense of Hörmander $[\mathbf{1 2}$, p. 56], then $Q$ is strictly convex at the relative boundary of $\omega$. If $Q$ is closed and there is a unbounded interval in $\mathbb{R}^{2 N-1}$ on which $f$ is affine then $Q$ is not strictly convex at its relative boundary.

2.5. Spaces of holomorphic functions. For an open set $D \subset \mathbb{C}^{N}$, we denote by $H(D)$ the space of all holomorphic functions on $D$ with its standard Fréchet topology. For a compact subset $K$ of $\mathbb{C}^{N}$, the space of all functions which are holomorphic on some open neighbourhood of $K$ is denoted by $H(K)$ and it is endowed with its natural inductive limit topology.

We denote by $H(Q)$ the vector space of all functions which are holomorphic on some open neighbourhood of the locally closed convex set $Q$. Let $\left(Q_{n}\right)_{n \in \mathbb{N}}$ be an increasing fundamental sequence of compact convex sets in $Q$. Since the algebraic equality $H(Q)=\cap_{n \in \mathbb{N}} H\left(Q_{n}\right)$ holds, we endow $H(Q)$ with the projective topology of $H(Q):=\operatorname{proj}_{n} H\left(Q_{n}\right)$. This topology does not depend of the choice of the fundamental system $\left(Q_{n}\right)_{n \in \mathbb{N}}$. See more details in [18, pp. 296-299]. In the case that $Q$ is a locally closed convex subset of $\mathbb{R}^{N}$, the space $H(Q)$ is a space 
of real analytic functions. In particular, if $Q$ is an open convex subset of $\mathbb{R}^{N}$, then $H(Q)=A(Q)$, where $A(Q)$ denotes the space of all real analytic functions on $Q$. The projective description of the Fourier Laplace transform of $H(Q)_{b}^{\prime}$ was studied by Ehrenpreis in $[\mathbf{9}]$ and by the authors in $[7]$.

2.6. Definition. For each set $D \subset \mathbb{C}^{N}$ we denote by $H_{D}: \mathbb{C}^{N} \rightarrow$ $\mathbb{R} \cup\{\infty\}$ the support function of $D: H_{D}(z):=\sup _{w \in D} \operatorname{Re}\langle z, w\rangle, z \in \mathbb{C}^{N}$. Here $\langle z, w\rangle:=\sum_{j=1}^{N} z_{j} w_{j}$. For each $n \in \mathbb{N}$ let $H_{n}:=H_{Q_{n}}$ be the support function of the convex compact set $Q_{n}$.

2.7. Problem of the projective description. Next, we recall the necessary notation for weighted inductive limits, see [3] and [4], and we state the problem of projective description. For notation concerning locally convex spaces we refer the reader to $[\mathbf{1 7}]$.

We denote by $V=\left(v_{n, k}\right)_{n, k \in \mathbb{N}}$ a double sequence of strictly positive upper semicontinuous weights on $\mathbb{C}^{N}, N \in \mathbb{N}$, such that

$$
v_{n+1, k}(z) \leq v_{n, k}(z) \leq v_{n, k+1}(z), \quad z \in \mathbb{C}^{N},
$$

for each $n, k \in \mathbb{N}$. The weighted inductive limit of Fréchet spaces $V H\left(\mathbb{C}^{N}\right)$ of entire functions associated with $V$ is defined by

$$
V H\left(\mathbb{C}^{N}\right):=\operatorname{ind}_{n \rightarrow \underset{ }{\operatorname{proj}}} H\left(v_{n, k}, \mathbb{C}^{N}\right),
$$

where the steps $H\left(v, \mathbb{C}^{N}\right)$ are defined, for a positive weight $v$ on $\mathbb{C}^{N}$, as the Banach space of entire functions

$$
H\left(v, \mathbb{C}^{N}\right):=\left\{f \in H\left(\mathbb{C}^{N}\right)\left|\|f\|_{v}:=\sup _{z \in \mathbb{C}^{N}} v(z)\right| f(z) \mid<\infty\right\} .
$$

The space $V H\left(\mathbb{C}^{N}\right)$ is a Hausdorff (LF)-space. In order to describe its topology by means of weighted sup-seminorms, Bierstedt, Meise, and Summers [3] associated with $V$ the system $\bar{V}$ of all those weights $\bar{v}: \mathbb{C}^{N} \rightarrow[0, \infty[$ which are upper semicontinuous and have the property that for each $n$ there are $\alpha_{n}>0$ and $k=k(n)$ such that $\bar{v} \leq \alpha_{n} v_{n, k}$ on $\mathbb{C}^{N}$. The projective hull of the weighted inductive limit is defined by $H \bar{V}\left(\mathbb{C}^{N}\right):=\left\{f \in H\left(\mathbb{C}^{N}\right)\left|\|f\|_{\bar{v}}:=\sup _{z \in \mathbb{C}^{N}} \bar{v}(z)\right| f(z) \mid<\infty\right.$ for all $\left.\bar{v} \in \bar{V}\right\}$, endowed with the Hausdorff locally convex topology defined by the system of seminorms $\left\{\|\cdot\|_{\bar{v}} \mid \bar{v} \in \bar{V}\right\}$. The projective hull is a complete locally convex space and $V H\left(\mathbb{C}^{N}\right)$ is contained in its projective hull with continuous inclusion. 
The problem of projective description is to determine conditions under which

(1) the spaces $V H\left(\mathbb{C}^{N}\right)$ and $H \bar{V}\left(\mathbb{C}^{N}\right)$ coincide algebraically, or

(2) the space $V H\left(\mathbb{C}^{N}\right)$ is a topological subspace of its projective hull $H \bar{V}\left(\mathbb{C}^{N}\right)$.

A positive answer to question (2), i.e., whether $V H\left(\mathbb{C}^{N}\right)$ is a topological subspace of its projective hull, is of particular importance, because when the answer is positive it permits to describe the topology of the weighted (LF)-space of holomorphic functions by means of weighted supseminorms.

In this article we are interested in the weight functions

$$
v_{n, k}(z):=\exp \left(-H_{n}(z)-|z| / k\right), \quad n, k \in \mathbb{N}, \quad z \in \mathbb{C}^{N},
$$

where $|z|:=\left(\sum_{j=1}^{N}\left|z_{j}\right|^{2}\right)^{1 / 2}$. By [18, Lemma 1.10], the Laplace transform

$$
\mathcal{F}(\varphi)(z):=\varphi(\exp \langle\cdot, z\rangle), \quad z \in \mathbb{C}^{N},
$$

is a linear topological isomorphism from the strong dual $H(Q)_{b}^{\prime}$ of $H(Q)$ onto $V H\left(\mathbb{C}^{N}\right)$.

We denote by $\bar{V}_{0}$ the set of all weights $\bar{v}$ such that there are unbounded increasing sequences $(k(n))_{n \in \mathbb{N}} \subset \mathbb{N}$ and $\left.\left(\alpha_{n}\right)_{n \in \mathbb{N}} \subset\right] 0, \infty[$ with

$$
\bar{v}(z)=\inf _{n \in \mathbb{N}} \exp \left(-H_{n}(z)-|z| / k(n)+\alpha_{n}\right) \text { for all } z \in \mathbb{C}^{N} .
$$

It is easy to see that every weight in $\bar{V}_{0}$ is contained in $\bar{V}$ and that every element in $\bar{V}$ is estimated by a weight in $\bar{V}_{0}$.

2.8. Remark. Let $G \subset \mathbb{C}^{N}$ be open and convex and let $\left(G_{n}\right)_{n \in \mathbb{N}}$ be a fundamental sequence of (convex) compact subsets $G_{n}$ of $G$ with $G_{n} \subset$ $\operatorname{int} G_{n+1}$ for all $n \in \mathbb{N}$. We put

$$
V_{G}:=\left(v_{G, n}\right)_{n \in \mathbb{N}}, \quad v_{G, n}(z):=\exp \left(-H_{G_{n}}(z)\right), \quad z \in \mathbb{C}^{N}, \quad n \in \mathbb{N} .
$$

By $[\mathbf{1 2}$, Theorem 4.7.3] the Laplace transform

$$
\mathcal{F}(\varphi)(z):=\varphi(\exp \langle\cdot, z\rangle), \quad z \in \mathbb{C}^{N},
$$

is a linear topological isomorphism from the strong dual $H(G)_{b}^{\prime}$ of $H(G)$ onto the weighted (LB)-space $V_{G} H\left(\mathbb{C}^{N}\right)$. Moreover, as a consequence of $\left[\mathbf{3}\right.$, Theorem 1.6], the space $V_{G} H\left(\mathbb{C}^{N}\right)$ and its projective hull $H \overline{V_{G}}\left(\mathbb{C}^{N}\right)$ coincide algebraically and topologically. 


\section{Spaces of holomorphic functions}

In this section we study the problem of topological projective description for the weighted (LF)-space $V H\left(\mathbb{C}^{N}\right)$.

3.1. Theorem. Let $Q \subset \mathbb{C}^{N}$ be a convex locally closed set. If $Q$ is strictly convex at the relative boundary of $\omega$, then the weighted inductive limit $V H\left(\mathbb{C}^{N}\right)$ coincides with its projective hull $H \bar{V}\left(\mathbb{C}^{N}\right)$ algebraically and topologically.

Proof: We first claim that the space $V H\left(\mathbb{C}^{N}\right)$ coincides algebraically and topologically with $\operatorname{proj}_{\leftarrow G \in \mathcal{G}} H(G)_{b}^{\prime}$, where $\mathcal{G}:=\{G \mid G$ is open and convex, $G \supset Q\}$. To show this as in the proof of [6, Theorem 6 (i)], note first that by [6, Proposition 2], we have $H(Q)=$ ind $_{\rightarrow G \in \mathcal{G}} H(G)$ algebraically. If $B$ is any bounded set in $H(Q)$ then $B$ is bounded in $H\left(Q_{n}\right)$ for each $n \in \mathbb{N}$. Since $H\left(Q_{n}\right)$ is a (DFS)-space, there exists a convex open neighbourhood $U_{n}$ of $Q_{n}$ so that $B \subset H\left(U_{n}\right)$ and such that the functions in $B$ are uniformly bounded on $U_{n}$. Then $V:=\bigcup_{n \in \mathbb{N}} U_{n}$ is an open neighbourhood of $Q$. Since $Q$ is strictly convex at the relative boundary of $\omega$, there is an open neighbourhood $U$ of $Q$ in $\mathcal{G}$ which is contained in $V$. Clearly, $B \subset H(U)$. From the construction it follows easily that $B$ is bounded in $H(U)$ and consequently bounded in ind $\rightarrow G \in \mathcal{G} H(G)$. Since $Q$ is strictly convex at the relative boundary of $\omega, H(Q)$ is bornological by results of Martineau [15] (see [18, Remark 1.9 (b)]). From this and the preceding considerations we get that $H(Q)=$ ind $\rightarrow G \in \mathcal{G} H(G)$ holds topologically and $H(Q)_{b}^{\prime}=\operatorname{proj}_{\leftarrow G \in \mathcal{G}} H(G)_{b}^{\prime}$. By Remark 2.8 this implies

$$
V H\left(\mathbb{C}^{N}\right)=\underset{\leftarrow G \in \mathcal{G}}{\operatorname{proj}} \underset{\leftarrow \bar{u} \in \bar{V}_{G}}{\operatorname{proj}} H\left(\bar{u}, \mathbb{C}^{N}\right) .
$$

Next we show that the space $H \bar{V}\left(\mathbb{C}^{N}\right)$ is contained in the space $\operatorname{proj}_{G} \operatorname{proj}_{\bar{u} \in \overline{V_{G}}} H\left(\bar{u}, \mathbb{C}^{N}\right)$ and that the inclusion is continuous. We fix a convex open neighbourhood $G$ of $Q$, a function $\kappa \in \overline{V_{G}}$ and show that there exists $\bar{v} \in \bar{V}$ with $\kappa \leq \bar{v}$. To do so, for every $n$ there is $\alpha_{n} \geq 1$ with $\kappa \leq \alpha_{n} \exp \left(-H_{G_{n}}\right)$. Since $G$ is a neighbourhood of $Q$, for each $n$ there exist $k(n)$ such that $Q_{n}+\frac{1}{k(n)} \bar{B}(0,1) \subset G_{k(n)}$ and, consequently, $H_{n}(z)+|z| / k(n) \leq H_{G_{k(n)}}(z)$ for all $z \in \mathbb{C}^{N}$. Hence

$$
\kappa(z) \leq \exp \left(-H_{n}(z)-|z| / k(n)+\log \alpha_{k(n)}\right) \text { for all } z \in \mathbb{C}^{N} \text { and } n \in \mathbb{N} \text {. }
$$

Moreover, the sequences $(k(n))_{n \in \mathbb{N}}$ and $\left(\alpha_{n}\right)_{n \in \mathbb{N}}$ can be taken increasing and unbounded. If we define

$$
\bar{v}(z):=\inf _{n \in \mathbb{N}} \exp \left(-H_{n}(z)-|z| / k(n)+\log \alpha_{k(n)}\right) \text { for all } z \in \mathbb{C}^{N},
$$


then $\bar{v} \in \bar{V}_{0}$ and $\kappa \leq \bar{v}$. From here it follows that $H \bar{V}\left(\mathbb{C}^{N}\right)$ is continuously embedded in $\operatorname{proj}_{G} \operatorname{proj}_{\bar{u} \in \overline{V_{G}}} H\left(\bar{u}, \mathbb{C}^{N}\right)$. Consequently, the space $V H\left(\mathbb{C}^{N}\right)$ and its projective hull $H \bar{V}\left(\mathbb{C}^{N}\right)$ coincide algebraically and topologically.

We prove in Theorem 3.11 below that the strict convexity of $Q$ at its relative boundary is necessary for $V H\left(\mathbb{C}^{N}\right)$ to be a topological subspace of $H \bar{V}\left(\mathbb{C}^{N}\right)$, if $Q$ has a basis of neighbourhoods which consists of domains of holomorphy. To show this we need some results about entire functions and differential operators of infinite order with constant coefficients.

3.2. Definition. An entire function $f$ on $\mathbb{C}^{N}$ is said to be of order at most one and zero type if for each $\varepsilon>0$ there is $C \geq 0$ with $|f(z)| \leq$ $C \exp (\varepsilon|z|)$ for all $z \in \mathbb{C}^{N}$. We denote by $A_{0}$ the set of all entire functions $f$ on $\mathbb{C}^{N}$ of order at most one and zero type. By [12, Theorem 4.7.3], $A_{0}=\mathcal{F}\left(H(\{0\})^{\prime}\right)$.

By [16, Lemme 15] each nonzero entire function $P \in A_{0}$ is slowly decreasing, i.e., for each $\varepsilon>0$ there is $R>0$ such that for all $z \in \mathbb{C}^{N}$ with $|z| \geq R$ there is $w \in \bar{B}(z, \varepsilon|z|)$ with $|P(w)| \geq \exp (-\varepsilon|w|)$. Here we denote $\bar{B}(\mu, r):=\left\{z \in \mathbb{C}^{N}|| z-\mu \mid \leq r\right\}, \mu \in \mathbb{C}^{N}, r \geq 0$.

We will use the following statements about functions in $A_{0}$.

3.3. Lemma. Let $P$ be a nonzero entire function on $\mathbb{C}^{N}$ of order at most one and zero type.

(i) For each locally bounded function $r: \mathbb{C}^{N} \rightarrow[0, \infty[$ satisfying $\lim _{|z| \rightarrow \infty} r(z) /|z|=0$, there is a continuous function $m: \mathbb{C}^{N} \rightarrow$ $\left[0, \infty\left[\right.\right.$ satisfying $\lim _{|z| \rightarrow \infty} m(z) /|z|=0$ and

$$
\sup _{|t-z| \leq r(z)}|P(t)| \leq \exp m(z) \text { for all } z \in \mathbb{C}^{N} .
$$

(ii) There exists a continuous function $r: \mathbb{C}^{N} \rightarrow[0, \infty[$ satisfying $\lim _{|z| \rightarrow \infty} r(z) /|z|=0$ and

$$
\sup _{|t-z| \leq r(z)}|P(t)| \geq \exp (-r(z)) \text { for all } z \in \mathbb{C}^{N} .
$$

Proof: (i) Since for each $\varepsilon>0$ there is $C>0$ such that $|P(z)| \leq$ $C \exp (\varepsilon|z|)$ for all $z \in \mathbb{C}^{N}$, for the function $\alpha(z):=\max (0 ; \log |P(z)|)$ 
we have $\lim _{|z| \rightarrow \infty} \alpha(z) /|z|=0$. From the inequalities

$$
\begin{aligned}
\left(\sup _{|t-z| \leq r(z)} \alpha(t)\right) /|z| & \leq \sup _{|t-z| \leq r(z)} \frac{|t|}{|z|} \sup _{|t-z| \leq r(z)} \frac{\alpha(t)}{|t|} \\
& \leq \frac{|z|+r(z)}{|z|} \sup _{|t| \geq|z|-r(z)} \frac{\alpha(t)}{|t|} \\
& =(1+r(z) /|z|) \sup _{|t| \geq|z|-r(z)} \frac{\alpha(t)}{|t|}
\end{aligned}
$$

and $\lim _{|z| \rightarrow \infty} r(z) /|z|=0, \lim _{|z| \rightarrow \infty}(|z|-r(z))=\infty$ it follows that $\beta(z):=\sup _{|t-z| \leq r(z)} \alpha(t)=o(|z|)$ as $|z| \rightarrow \infty$. As $m$ we can choose any continuous majorant of $\beta$ with $m(z)=o(|z|)$ as $|z| \rightarrow \infty$.

(ii) Since $P$ is of order at most one and zero type, we can select an increasing unbounded sequence $R_{n}>0$ such that for each $n \in \mathbb{N}$ and each $z \in \mathbb{C}^{N}$ with $|z| \geq R_{n}$ there exists $w \in \bar{B}(z,|z| / n)$ such that $|P(w)| \geq \exp (-|w| / n)$. We put $s(z):=1$ if $|z|<R_{2}$ and $s(z):=|z| / n$ if $R_{n+1} \leq|z|<R_{n+2}$ and $n \in \mathbb{N}$.

For $z \in \mathbb{C}^{N}$ with $|z| \in\left[R_{n+1}, R_{n+2}\right), n \in \mathbb{N}$, we choose $w \in \bar{B}(z,|z| /(n+$ 1)) $\subset \bar{B}(z, s(z))$ such that $|P(w)| \geq \exp (-|w| /(n+1))$. Then

$$
\begin{aligned}
\sup _{|t-z| \leq s(z)}|P(t)| & \geq \exp \left(-\frac{|z|+|z| /(n+1)}{n+1}\right)=\exp \left(-\frac{n+2}{(n+1)^{2}}|z|\right) \\
& \geq \exp \left(-\frac{|z|}{n}\right)=\exp (-s(z)) .
\end{aligned}
$$

As $r$ we can choose any continuous majorant of $s$ with $\lim _{|z| \rightarrow \infty} r(z) /|z|=$ 0 .

3.4. Lemma. (i) For each $\bar{v} \in \bar{V}$ and every upper semicontinuous function $m: \mathbb{C}^{N} \rightarrow \mathbb{R}$ such that $\lim _{|z| \rightarrow \infty} m(z) /|z|=0$ the function $\bar{v} \exp m$ belongs also to $\bar{V}$.

(ii) For each $\bar{v} \in \bar{V}$ and every locally bounded function $r(z): \mathbb{C}^{N} \rightarrow$ $\left[0, \infty\left[\right.\right.$ for which $\lim _{|z| \rightarrow \infty} r(z) /|z|=0$ there is $\bar{u} \in \bar{V}$ with $\bar{v} \leq$ $\inf _{|t-z| \leq r(z)} \bar{u}(t)$ for all $z \in \mathbb{C}^{N}$.

Proof: (i) This follows easily from the definition of $\bar{V}$ and the condition on $m$.

(ii) It is enough to show the assertion for $\bar{v} \in \bar{V}_{0}$. To do so, fix

$$
\bar{v}(z)=\inf _{n \in \mathbb{N}} \exp \left(-H_{n}(z)-|z| / k(n)+\alpha_{n}\right) \in \bar{V}_{0} .
$$


Since for all $n \in \mathbb{N}$ and $\varepsilon>0$ the function $H_{n}(z)+\varepsilon|z|$ is subadditive and positively homogeneous, for each $n \in \mathbb{N}$ there exists $C_{n} \geq 0$ such that

$\sup _{|t-z| \leq r(z)}\left(H_{n}(t)+|t| /(k(n)+1)\right) \leq H_{n}(z)+|z| / k(n)+C_{n}$ for all $z \in \mathbb{C}^{N}$

Moreover, the sequence $\left(C_{n}\right)_{n \in \mathbb{N}}$ can be taken increasing. The desired function is

$$
\bar{u}(z):=\inf _{n} \exp \left(-H_{n}(z)-|z| /(k(n)+1)+\alpha_{n}+C_{n}\right), \quad z \in \mathbb{C}^{N} .
$$

3.5. Lemma. For each nonzero entire function $P \in A_{0}$ the multiplication operator

$$
M_{P}: V H\left(\mathbb{C}^{N}\right) \rightarrow V H\left(\mathbb{C}^{N}\right), \quad M_{P}(f):=P f,
$$

is an injective topological homomorphism if $V H\left(\mathbb{C}^{N}\right)$ is endowed with the topology induced by $H \bar{V}\left(\mathbb{C}^{N}\right)$.

Proof: It is clear that $M_{P}$ is injective and linear. To show that $M_{P}$ is continuous we fix a function $\bar{v} \in \bar{V}$. By Lemma 3.3 (i) there is a continuous function $m: \mathbb{C}^{N} \rightarrow\left[0, \infty\left[\right.\right.$ such that $\lim _{|z| \rightarrow \infty} m(z) /|z|=0$ and $|P(z)| \leq \exp (m(z))$ for all $z \in \mathbb{C}^{N}$. Then $\bar{u}:=\bar{v} \exp m \in \bar{V}$ and for all $f \in V H\left(\mathbb{C}^{N}\right)$

$$
\left\|M_{P}(f)\right\|_{\bar{v}}=\sup _{z \in \mathbb{C}^{N}}|P(z) f(z)| \bar{v}(z) \leq\|f\|_{\bar{u}} .
$$

Consequently, $M_{P}: V H\left(\mathbb{C}^{N}\right) \rightarrow V H\left(\mathbb{C}^{N}\right)$ is continuous if $V H\left(\mathbb{C}^{N}\right)$ is endowed with the topology induced by $H \bar{V}\left(\mathbb{C}^{N}\right)$.

To show that the division map $P f \mapsto f$ is continuous for this topology, choose a continuous function $r: \mathbb{C}^{N} \rightarrow[0, \infty$ [ according to Lemma 3.3 (ii) and a continuous function $m: \mathbb{C}^{N} \rightarrow[0, \infty[$ for $4 r$ according to Lemma 3.3 (i). Fix $\bar{v} \in \bar{V}$. By Lemma 3.4 (i) for $m+2 r$, the function $\bar{v} \exp (m+2 r)$ belongs to $\bar{V}$, and we can apply Lemma 3.4 (ii) to conclude that there is $\bar{w} \in \bar{V}$ such that

$$
\bar{v}(z) \exp (m(z)+2 r(z)) \leq \inf _{|t-z| \leq 4 r(z)} \bar{w}(t) .
$$


By Hörmander's division lemma [11, Lemma 3.2], for each $f \in V H\left(\mathbb{C}^{N}\right)$ and $z \in \mathbb{C}^{N}$, we have

$$
\begin{aligned}
|f(z)| & =\left|\frac{(P f)(z)}{P(z)}\right| \\
& \leq \frac{|t-z| \leq 4 r(z)}{\left.\sup _{|t-z| \leq r(z)}|P(t) f(t)| \sup _{|t-z| \leq 4 r(z)}|P(t)|\right)^{2}} \\
& \leq\|P f\|_{\bar{w}}\left(\sup _{|t-z| \leq 4 r(z)} \bar{w}(t)^{-1}\right) \exp (m(z)) \exp (2 r(z))
\end{aligned}
$$

and consequently,

$$
|f(z)| \bar{v}(z) \leq\|P f\|_{\bar{w}} \frac{\bar{v}(z) \exp (m(z)+2 r(z))}{\inf _{|t-z| \leq 4 r(z)} \bar{w}(t)} .
$$

Hence $|f|_{\bar{v}} \leq|P f|_{\bar{w}}$ for each $f \in V H\left(\mathbb{C}^{N}\right)$. The proof is complete.

Next, we recall the necessary notations for differential operators of infinite order.

3.6. Definition. Let $P(z):=\sum_{\alpha \in \mathbb{N}_{0}^{N}} a_{\alpha} z^{\alpha}$ be an entire function on $\mathbb{C}^{N}$ of order at most one and zero type. By Gol'dberg's theorem in $[\mathbf{2 0}$, Theorem 3.1.1] an entire function $P(z)=\sum_{\alpha \in \mathbb{N}_{0}^{N}} a_{\alpha} z^{\alpha}$ is of at most order one and zero type if and only if $\lim _{|\alpha| \rightarrow \infty}|\alpha|\left|a_{\alpha}\right|^{1 /|\alpha|}=0$.

For each open set $G$ in $\mathbb{C}^{N}$ and every function $f \in H(G)$ the series $P(D) f:=\sum_{\alpha \in \mathbb{N}_{0}^{N}} a_{\alpha} f^{(\alpha)}$ converges absolutely in the space $H(G)$ and the linear differential operator $P(D)$ maps $H(G)$ continuously into $H(G)$ (see [16]). Consequently, $P(D)$ is a continuous linear operator from $H(Q)$ into $H(Q)$.

For each $\lambda \in \mathbb{C}^{N}$ the equality $P(D)(\exp \langle\cdot, \lambda\rangle)=P(\lambda) \exp \langle\cdot, \lambda\rangle$ holds.

If $P(D)^{t}: H(Q)^{\prime} \rightarrow H(Q)^{\prime}$ is the adjoint operator to $P(D)$, then

$$
\mathcal{F} \circ P(D)^{t} \circ \mathcal{F}^{-1}=M_{P},
$$

where $M_{P}$ is the multiplication operator $f \mapsto P f$ from $V H\left(\mathbb{C}^{N}\right)$ into $V H\left(\mathbb{C}^{N}\right)$.

3.7. Remark. Let $P(D)$ be a differential operator of infinite order and $U$ a unitary transformation of $\mathbb{C}^{N}$ with the inverse $U^{-1}$. The set $U(Q)$ is convex and locally closed. The map $T_{U}(f):=f \circ U$ is a topological isomorphism of $H(U(Q))$ onto $H(Q)$. 
The map $T_{U} \circ P(D) \circ T_{U^{-1}}$ is also a differential operator of infinite order.

Proof: Let $\left(U^{-1}\right)^{t}$ be the map defined by the transpose of the matrix of $U^{-1}$. It is easy to check from the definition that the function $P_{U^{-1}}:=$ $P \circ\left(U^{-1}\right)^{t}$ belongs also to $A_{0}$. For each $\lambda \in \mathbb{C}^{N}$ we have, for $e_{\lambda}:=$ $\exp \langle\cdot, \lambda\rangle$,

$$
\begin{aligned}
T_{U} \circ P(D) \circ T_{U^{-1}}\left(e_{\lambda}\right) & =T_{U} \circ P(D)\left(T_{U^{-1}}\left(e_{\lambda}\right)\right) \\
& =T_{U} \circ P(D)\left(e_{\left(U^{-1}\right)^{t}(\lambda)}\right) \\
& =T_{U}\left(P\left(\left(U^{-1}\right)^{t}(\lambda)\right) e_{\left.U^{-1}\right)^{t}(\lambda)}\right) \\
& =P\left(\left(U^{-1}\right)^{t}(\lambda)\right) e_{\left.U^{t}\left(U^{-1}\right)^{t}(\lambda)\right)} \\
& =P\left(\left(U^{-1}\right)^{t}(\lambda)\right) e_{\lambda}=P_{U^{-1}}(\lambda) e_{\lambda} \\
& =P_{U^{-1}}(D) e_{\lambda} .
\end{aligned}
$$

Since the system $\left\{e_{\lambda} \mid \lambda \in \mathbb{C}^{N}\right\}$ is total in $H(Q)$, the continuous linear operators $P_{U^{-1}}(D)$ and $T_{U} \circ P(D) \circ T_{U^{-1}}$ coincide on $H(Q)$.

The notation below has to be introduced, as we need Theorem 3.9 about the surjectivity of a convolution operator on the spaces of holomorphic functions on convex locally closed sets in $\mathbb{C}$.

3.8. Definition. Let $B$ be a convex compact set in $\mathbb{C}, \mu \in H(B)^{\prime}$ and $G$ a convex and locally closed set in $\mathbb{C}$. We denote by $a$ the Laplace transform of $\mu$ and define the convolution operator $L_{a}: H(G+B) \rightarrow$ $H(G)$ by

$$
L_{a}(f)(z):=\mu(f(z+\cdot)) .
$$

Here the spaces $H(G+B)$ and $H(G)$ are endowed with their natural inductive limit topologies. (By [15, Theorem 1.23] (see also [18, Remarks 1.5 and 1.9]) the inductive limit topologies in $H(G+B)$ and $H(G)$ coincide with the corresponding projective topologies.) The operator $L_{a}$ is linear and continuous from $H(G+B$ ) into $H(G)$ (see [18]).

We apply later Theorem 3.9 for a special case: If $a(z)=\sum_{\alpha \in \mathbb{N}_{0}} a_{\alpha} z^{\alpha} \in$ $A_{0}$, then $B=\{0\}$ and the convolution operator $L_{a}$ is the differential operator $a(D)$.

Let $\Lambda^{*}$ be the set of all accumulation points of $\{-\arg \lambda \mid \lambda \in \mathbb{C}, a(\lambda)=$ $0\}$. For $z \in \mathbb{C}, \varphi \in \mathbb{R}$ we define a ray

$$
H_{z}^{+}(\varphi):=\left\{w \in \mathbb{C} \mid \operatorname{Re}\left((w-z) e^{-i \varphi}\right)=0 \text { and } \operatorname{Im}\left((w-z) e^{-i \varphi}\right)>0\right\} .
$$


By [14], $\varphi \in \mathbb{R}$ is called a direction of right-hand quasinonconvexity of $G$ if there is $z \in G \cap(\partial G)$ such that either there exists $w \in((\partial G) \backslash G) \cap$ $H_{z}^{+}(\varphi)$ with $[z, w] \subset \partial G$ or $H_{z}^{+}(\varphi) \subset G \cap(\partial G)$. Using $H_{z}^{+}(\varphi+\pi)$ instead of $H_{z}^{+}(\varphi)$ we get the definition of a direction $\varphi \in \mathbb{R}$ of left-hand quasinonconvexity of $G$.

The following result is a partial case of the Theorem 3 of $[\mathbf{1 4}]$. For an interval $G$ this statement was obtained also by Napalkov, Rudakov [19] and Korobeinik [13].

3.9. Theorem. If the operator $L_{a}: H(G+B) \rightarrow H(G)$ is surjective, then there is a $2 \pi$-periodic function $\gamma: \mathbb{R} \rightarrow(0, \pi / 2)$ such that $(\nu-$ $\gamma(\nu), \nu) \cap \Lambda^{*}=\emptyset$ for each direction $\nu \in \mathbb{R}$ of left-hand quasinonconvexity of $G$ and $(\nu, \nu+\gamma(\nu)) \cap \Lambda^{*}=\emptyset$ for each direction $\nu \in \mathbb{R}$ of right-hand quasinonconvexity of $G$.

The following statement was proved in [18, Proposition 1.16] under the assumption that $Q$ is $\mathbb{C}$-strictly convex at the relative boundary of $\omega$. Its proof in this article is essentially the same as in [18]. We give the details for the convenience of the reader.

3.10. Proposition. Suppose that $Q \subset \mathbb{C}^{N}$ is convex, locally closed and has a neighbourhood basis of domains of holomorphy. If each nonzero differential operator $P(D): H(Q) \rightarrow H(Q)$ is surjective then $Q$ is strictly convex at the relative boundary of $\omega$.

Proof: To argue by contradiction we treat the case $N=1$ first. If $Q \subset \mathbb{C}$ is locally closed and not strictly convex at the relative boundary of $\omega$, then there is a supporting line $R$ for $\bar{Q}$ such that $I:=R \cap Q$ is not compact. After a shift and a rotation we may assume that $R$ is the real line and that zero is an interior point of $I$. By our assumption, one of the sets $I_{ \pm}:=I \cap\{z \in \mathbb{C} \mid \pm \operatorname{Re} z>0\}$ is not compact. Without restriction we assume that $I_{+}$is not compact. Then we choose a sequence $\left(z_{n}\right)_{n \in \mathbb{N}}$ in $\mathbb{C}$ in such a way that $\sum_{n \in \mathbb{N}}\left|z_{n}\right|^{-1}$ converges and that for each $k \in \mathbb{N}$ the set $\left\{n \in \mathbb{N} \mid \arg z_{n}=-\frac{\pi}{2}+\frac{1}{k}\right\}$ is infinite. Then the function $P: \mathbb{C} \rightarrow$ $\mathbb{C}$, defined by $P(z):=\prod_{n \in \mathbb{N}}\left(1-z / \bar{z}_{n}\right)$ is an entire function of order at most one and zero type. Now note that in the notation of Definition 3.8 we have $H_{0}^{+}(-\pi / 2) \cap I=I_{+}$. Hence our assumption on $I_{+}$implies that $-\pi / 2$ is a direction of right hand quasiconvexity of $Q$. By the choice of $\left(z_{n}\right)_{n \in \mathbb{N}}$, the set $\Lambda^{*}$ of all accumulation points of $\{-\arg \lambda \mid P(\lambda)=0\}$ contains $\left\{-\frac{\pi}{2}+\frac{1}{k} \mid k \in \mathbb{N}\right\}$. Consequently, $\Lambda^{*} \cap(-\pi / 2,-\pi / 2+\varepsilon) \neq \emptyset$ for each $\varepsilon>0$. By Theorem 3.9, this implies that the operator $P(D)$ is not surjective on $H(Q)$. This proves the proposition for $N=1$. 
To complete the proof we assume now that $N \geq 2$ and that there is a supporting hyperplane $R$ for $\bar{Q}$ such that $R \cap Q$ is not compact. Since $R \cap Q$ is convex, after a shift, we can assume that there is a real line $S$ in $\mathbb{C}^{N}$ such that 0 is an interior point of $S \cap R \cap G$ and such that $S \cap R \cap G$ is not compact. Choosing appropriate coordinates, we may assume that the complex line generated by $S$ is $\mathbb{C} \times\{0\}$. Then $Q^{\prime}:=Q \cap(\mathbb{C} \times\{0\})$ can be considered as a subset of $\mathbb{C}$. Obviously, $Q^{\prime}$ is convex and locally closed but not strictly convex at the relative boundary of $\omega^{\prime}:=Q^{\prime} \cap \partial_{r} Q^{\prime}$. Hence the case $N=1$ implies the existence of a differential operator $P\left(\frac{\partial}{\partial z_{1}}\right)$ on $H\left(Q^{\prime}\right)$ which is not surjective. In particular, we can choose $g \in H\left(Q^{\prime}\right)$ which is not in the range of $P\left(\frac{\partial}{\partial z_{1}}\right)$. By the definition of $H\left(Q^{\prime}\right)$ there exists an open neighbourhood $\Omega^{\prime}$ in $\mathbb{C}$ of $Q^{\prime}$ such that $g$ is in $H\left(\Omega^{\prime}\right)$. Now we use the hypothesis to choose a domain of holomorphy $X$ which is a neighbourhood of $Q$ and satisfies $X \cap(\mathbb{C} \times\{0\}) \subset \Omega^{\prime} \times\{0\}$. Since $X \cap(\mathbb{C} \times\{0\})$ is a closed subvariety of $X$ it follows from [12, Notions 4.2.12] that there exists $G \in H(X)$ such that $G\left(z_{1}, 0\right)=g\left(z_{1}\right)$ for each $\left(z_{1}, 0\right) \in X_{0}:=X \cap(\mathbb{C} \times\{0\})$. Now note that for each $F \in H(Q)$ which satisfies $P(D) F=G$ we have $P\left(\frac{\partial}{\partial z_{1}}\right)\left(\left.F\right|_{X_{0}}\right)=g$ in contradiction to the choice of $g$. Hence $P(D)$ is not surjective on $H(Q)$. By contradiction, this completes the proof.

3.11. Theorem. Let $Q \subset \mathbb{C}^{N}$ be a convex locally closed set. Suppose that $Q$ has a neighbourhood basis of domains of holomorphy. If $V H\left(\mathbb{C}^{N}\right)$ is a topological subspace of $H \bar{V}\left(\mathbb{C}^{N}\right)$, then $Q$ is strictly convex at the relative boundary of $\omega$.

Proof: Suppose that $V H\left(\mathbb{C}^{N}\right)$ is a topological subspace of $H \bar{V}\left(\mathbb{C}^{N}\right)$. By Lemma 3.5 for each nonzero entire function $P \in A_{0}$ the multiplication operator $M_{P}=P(D)^{t}: V H\left(\mathbb{C}^{N}\right) \rightarrow V H\left(\mathbb{C}^{N}\right)$ is an injective topological homomorphism. Since the space $H(Q)$ is reflexive, an application of the Hahn-Banach theorem gives that $P(D): H(Q) \rightarrow H(Q)$ is surjective for each such $P$. By Proposition 3.10, $Q$ is strictly convex at the relative boundary of $\omega$.

3.12. Remark. (a) If a convex and locally closed set $Q \subset \mathbb{C}^{N}$ is $\mathbb{C}$ strictly convex at the relative boundary of $\omega$ (for example this is the case if $N=1$ ) then $Q$ has a neighbourhood basis of domains of holomorphy. In fact, by [15, Lemme 3 of the proof of Théorème 1.2] (see also the proof of Melikhov, Momm [18, Proposition 1.16]) if $Q$ is $\mathbb{C}$-strictly convex at the relative boundary of $\omega$, then $Q$ has a neighbourhood basis of linearly convex open sets, hence a basis of domains of holomorphy. An 
open convex set in $\mathbb{C}^{N}$ is linearly convex if its complement is a union of complex hyperplanes.

(b) If $Q$ is a convex and locally closed subset of $\mathbb{R}^{N}$ then also $Q$ has a neighbourhood basis of domains of holomorphy.

In fact, let $U$ be an open neighbourhood of $Q$ in $\mathbb{C}^{N}$. By a lemma of Cartan [8, Proposition 1], $U \cap \mathbb{R}^{N}$ has a neighbourhood basis $\mathcal{U}$ of domains of holomorphy. Hence there exists $V \in \mathcal{U}$ such that $V \subset U$. Since $Q \subset U \cap \mathbb{R}^{N} \subset V$ then $V$ is a neighbourhood of $Q$.

As in [6, Corollary 7 (a)], on account of Remark 3.12 (b), we obtain the following statement.

3.13. Corollary. Let $Q$ be a convex subset of $\mathbb{R}^{N}$ which is locally closed. The weighted inductive limit $V H\left(\mathbb{C}^{N}\right)$ is a topological subspace of its projective hull $H \bar{V}\left(\mathbb{C}^{N}\right)$ if and only if $Q$ is compact.

Problem. Which locally closed convex sets $Q$ have a neighbourhood basis of domains of holomorphy?

A necessary and sufficient condition for the algebraic equality $V H(\mathbb{C})=H \bar{V}(\mathbb{C})$ in the case of a bounded convex locally closed set $Q$ in $\mathbb{C}$ is presented in $\left[6\right.$, Theorem 8]. The algebraic identity $V H\left(\mathbb{C}^{N}\right)=$ $H \bar{V}\left(\mathbb{C}^{N}\right)$ also holds in case $Q$ is a convex open subset of $\mathbb{R}^{N}$ as it was proved in [7, Theorem 3.4]. This is the case of the Fourier Laplace transform of the space of analytic functionals. The characterization of the algebraic identity $V H\left(\mathbb{C}^{N}\right)=H \bar{V}\left(\mathbb{C}^{N}\right)$ for a general locally closed convex set $Q$ remains open. We plan to return to this question in the future.

\section{Spaces of continuous functions}

The weighted (LF)-space of continuous functions $V C\left(\mathbb{C}^{N}\right)$ and its projective hull $C \bar{V}\left(\mathbb{C}^{N}\right)$ associated with the sequence $V=\left(v_{n, k}\right)_{n, k \in \mathbb{N}}$ of Section 2 are defined by replacing entire functions by continuous ones:

$$
\begin{aligned}
& C V_{n}\left(\mathbb{C}^{N}\right):=\left\{f \in C\left(\mathbb{C}^{N}\right) \mid\|f\|_{k}\right. \\
&\left.:=\sup _{z \in \mathbb{C}^{N}} v_{n, k}(z)|f(z)|<\infty \text { for all } k \in \mathbb{N}\right\}, \quad n \in \mathbb{N} ; \\
& V C\left(\mathbb{C}^{N}\right):=\operatorname{ind}_{n \rightarrow} C V_{n}\left(\mathbb{C}^{N}\right) ; \\
& C \bar{V}\left(\mathbb{C}^{N}\right):=\left\{f \in C\left(\mathbb{C}^{N}\right)\left|\|f\|_{\bar{v}}:=\sup _{\bar{v} \in \bar{V}} v(z)\right| f(z) \mid<\infty \text { for all } \bar{v} \in \bar{V}\right\} .
\end{aligned}
$$


Clearly $V H\left(\mathbb{C}^{N}\right)$ is continuously included in $V C\left(\mathbb{C}^{N}\right)$ and $H \bar{V}\left(\mathbb{C}^{N}\right)$ is topological subspace of $C \bar{V}\left(\mathbb{C}^{N}\right)$. The problem of topological projective description for spaces of continuous functions has a positive answer, as we show next.

4.1. Theorem. For every locally closed convex set $Q \subset \mathbb{C}^{N}$ the weighted (LF)-space $V C\left(\mathbb{C}^{N}\right)$ is a topological subspace of its projective hull $C \bar{V}\left(\mathbb{C}^{N}\right)$.

Proof: For each $n$ the sequence $V_{n}:=\left(v_{n, k}\right)_{k \in \mathbb{N}}$ is increasing and $\lim _{|z| \rightarrow \infty} v_{n, k}(z) / v_{n, k+1}(z)=\infty$ for all $k$. Hence for every $n$ the weighted Fréchet space $C V_{n}\left(\mathbb{C}^{N}\right)$ coincides with the Fréchet space $C\left(V_{n}\right)_{0}\left(\mathbb{C}^{N}\right):=\left\{f \in C\left(\mathbb{C}^{N}\right)\left|v_{n, k}\right| f \mid\right.$ vanishes at infinity for all $\left.k \in \mathbb{N}\right\}$. By [3, Theorem 1.3] $V C\left(\mathbb{C}^{N}\right)$ is a topological subspace of its projective hull $C \bar{V}\left(\mathbb{C}^{N}\right)$.

To investigate the problem of algebraic projective description for spaces of continuous functions we observe first that, by [2, Proposition 2.4 and Theorem 2.7], the algebraic equality $V C\left(\mathbb{C}^{N}\right)=C \bar{V}\left(\mathbb{C}^{N}\right)$ is equivalent to the following condition $(\mathrm{wQ})$ on the weights, which was originally introduced by $\operatorname{Vogt}[\mathbf{2 1}$, Section 5$]$; see also $[\mathbf{2}, \S 2$, Definition 1]:

i.e.,

$$
\begin{gathered}
\forall n \in \mathbb{N} \exists m \geq n, k \in \mathbb{N} \forall \mu \geq m, l \in \mathbb{N} \exists L \in \mathbb{N}, C>0 \\
v_{m, l} \leq C \max \left(v_{n, k}, v_{\mu, L}\right),
\end{gathered}
$$

$$
\begin{aligned}
& \exp \left(-H_{m}(z)-|z| / l\right) \\
& \quad \leq C \max \left(\exp \left(-H_{n}(z)-|z| / k\right), \exp \left(-H_{\mu}(z)-|z| / L\right)\right), \quad z \in \mathbb{C}^{N} .
\end{aligned}
$$

Let $S:=\left\{z \in \mathbb{C}^{N}|| z \mid=1\right\}$.

4.2. Lemma. The condition $(w Q)$ (i.e., (4.1)) is equivalent to

$$
\forall n \in \mathbb{N} \exists m \geq n, k \in \mathbb{N} \forall \mu \geq m, l \in \mathbb{N} \exists L \in \mathbb{N} \forall a \in S
$$

$$
H_{m}(a)+\frac{1}{l} \geq \min \left(H_{n}(a)+\frac{1}{k}, H_{\mu}(a)+\frac{1}{L}\right) .
$$

Proof: Condition (4.2) implies condition (4.1) since support functions are positively homogeneous.

Suppose that condition (4.1) holds. Then

$\forall n \in \mathbb{N} \exists m \geq n, k \in \mathbb{N} \forall \mu \geq m, l \in \mathbb{N} \exists L \in \mathbb{N}, C>0 \forall t>0, a \in S$

$$
t H_{m}(a)+t / l \geq-\log C+\min \left(t H_{n}(a)+t / k, t H_{\mu}(a)+t / L\right),
$$


hence

$$
H_{m}(a)+1 / l \geq-(\log C) / t+\min \left(H_{n}(a)+1 / k, H_{\mu}(a)+1 / L\right) .
$$

Letting $t \rightarrow \infty$, we obtain (4.2).

4.3. Notation. The convex hull of a set $D \subset \mathbb{C}^{N}$ is denoted by $\operatorname{conv}(D)$. Let $\left(\omega_{n}\right)_{n \in \mathbb{N}}$ be a compact exhaustion of $\omega$ such that $\omega_{n} \subset \omega_{n+1}$ for all $n \in \mathbb{N}$. We can suppose that $Q_{n}=\operatorname{conv}\left(\omega_{n} \cup K_{n}\right)$, where $\left(K_{n}\right)_{n \in \mathbb{N}}$ is a fundamental system of (convex) compact subsets of int ${ }_{r} Q$ with $K_{n} \subset$ $K_{n+1}$ for each $n \in \mathbb{N}$; see [18, Lemma 1.2]. We define the following sets of supporting directions:

$$
\begin{aligned}
& S_{\omega_{n}}:=\left\{a \in S \mid \exists z \in \omega_{n}: \operatorname{Re}\langle z, a\rangle=H_{Q}(a)\right\}, \quad n \in \mathbb{N}, \\
& S_{\omega}:=\left\{a \in S \mid \exists z \in \omega: \operatorname{Re}\langle z, a\rangle=H_{Q}(a)\right\} .
\end{aligned}
$$

If int $Q \neq \emptyset$, then for $a \in S$ and $n \in \mathbb{N}$ the equality $H_{n}(a)=H_{Q}(a)$ holds if and only if $a \in S_{\omega_{n}}$. Indeed, let $a \in S_{\omega_{n}}$. Then there is $z \in \omega_{n}$ with $H_{Q}(a)=\operatorname{Re}\langle z, a\rangle$. From $\omega_{n} \subset Q_{n} \subset Q$ it follows that $\operatorname{Re}\langle z, a\rangle \leq$ $H_{\omega_{n}}(a) \leq H_{n}(a) \leq H_{Q}(a)$ and $H_{n}(a)=H_{Q}(a)$. Conversely, assume that $H_{n}(a)=H_{Q}(a)$. Since $Q_{n}=\operatorname{conv}\left(K_{n} \cup \omega_{n}\right)$ the equality $H_{n}(a)=$ $\max \left\{H_{K_{n}}(a), H_{\omega_{n}}(a)\right\}$ holds. The inclusion $K_{n} \subset \operatorname{int} Q$ implies that $H_{K_{n}}(b)<H_{Q}(b)$ for each $b \in S$. Consequently $H_{\omega_{n}}(a)=H_{Q}(a)$. Since the set $\omega_{n}$ is compact there exists $z \in \omega_{n}$ such that $H_{\omega_{n}}(a)=$ sup $\operatorname{Re}\langle t, a\rangle=\operatorname{Re}\langle z, a\rangle$. Hence $H_{Q}(a)=\operatorname{Re}\langle z, a\rangle$ and $a \in S_{\omega_{n}}$. $t \in \omega_{n}$

For a set $A \subset S$ we put as in [18]

$$
F_{A}:=\left\{z \in \omega \mid \exists a \in A: \operatorname{Re}\langle z, a\rangle=H_{Q}(a)\right\} .
$$

The following lemma was obtained in [18, Lemmas 3.4 and 3.5] for a bounded convex locally closed set $Q \subset \mathbb{C}^{N}$.

4.4. Lemma. If a convex locally closed set $Q \subset \mathbb{C}^{N}$ is strictly convex at the relative boundary of $\omega$, then the following assertions hold:

(i) The set $S_{\omega}$ is open in $S$.

(ii) If $A \subset S_{\omega}$ is compact then $F_{A}$ is also compact.

(iii) $\left(S_{\omega_{n}}\right)_{n \in \mathbb{N}}$ is a compact exhaustion of $S_{\omega}$.

Proof: If int $Q=\emptyset$, then $Q$ is compact, $\omega=\partial_{r} Q$ and $S_{\omega}=S$. Obviously the assertions (i), (ii) and (iii) hold.

If int $Q$ is not empty then we argue as follows:

(i): If $\omega=\partial Q$ then $S_{\omega}=S$ and $S_{\omega}$ is open in $S$. 
Let $\omega \neq \partial Q$. We fix $a \in S_{\omega}$ and $z \in \omega$ with $\operatorname{Re}\langle z, a\rangle=H_{Q}(a)$. We will prove first that there is a neighbourhood $U$ of $a$ in $S$ such that

$$
\sup _{c \in U} \sup _{w \in(\partial Q) \backslash \omega} \operatorname{Re}\langle w, c\rangle<H_{Q}(a) .
$$

We suppose the contrary. Then there are $a_{n} \in S, w_{n} \in(\partial Q) \backslash \omega$ such that $\lim _{n \rightarrow \infty} a_{n}=a$ and

$$
\operatorname{Re}\left\langle a_{n}, w_{n}\right\rangle \geq H_{Q}(a)-\frac{1}{n} \text { for all } n \in \mathbb{N} .
$$

Assume that $\left(w_{n}\right)_{n \in \mathbb{N}}$ is bounded. We choose a subsequence $\left(w_{n_{s}}\right)_{s \in \mathbb{N}}$ and $w \in \partial Q$ with $\lim _{s \rightarrow \infty} w_{n_{s}}=w$. Since the set $(\partial Q) \backslash \omega$ is closed then $w \in(\partial Q) \backslash \omega$. From (4.4) it follows that $\operatorname{Re}\langle a, w\rangle \geq H_{Q}(a)$ and consequently $\operatorname{Re}\langle a, w\rangle=H_{Q}(a)$. Hence $[z, w] \subset \partial Q$ but $w \notin Q$. This contradicts the strict convexity of $Q$ at the relative boundary of $\omega$.

Let now $\left(w_{n}\right)_{n \in \mathbb{N}}$ be unbounded. We select a subsequence $\left(w_{n_{s}}\right)_{s \in \mathbb{N}}$ with $\lim _{s \rightarrow \infty}\left|w_{n_{s}}\right|=\infty$. Without loss of generality we can assume that the sequence $v_{s}:=\left|w_{n_{s}}-z\right|^{-1}\left(w_{n_{s}}-z\right), s \in \mathbb{N}$, converges to $b \in S$. Since $\bar{Q}$ is convex, for each $\alpha>0$ and for all $s \in \mathbb{N}$ such that $\alpha<\left|w_{n_{s}}-z\right|$ the point $z_{\alpha, s}:=z+\alpha v_{s}$ belongs to $\bar{Q}$. Hence $z+\alpha b \in \bar{Q}$ for all $\alpha>0$. Note that, by (4.4),

$$
\begin{aligned}
\operatorname{Re}\left\langle a_{n_{s}}, z_{\alpha, s}\right\rangle & =\left(1-\frac{\alpha}{\left|w_{n_{s}}-z\right|}\right) \operatorname{Re}\left\langle a_{n_{s}}, z\right\rangle+\frac{\alpha}{\left|w_{n_{s}}-z\right|} \operatorname{Re}\left\langle a_{n_{s}}, w_{n_{s}}\right\rangle \\
& \geq\left(1-\frac{\alpha}{\left|w_{n_{s}}-z\right|}\right) \operatorname{Re}\left\langle a_{n_{s}}, z\right\rangle+\frac{\alpha}{\left|w_{n_{s}}-z\right|}\left(H_{Q}(a)-\frac{1}{n_{s}}\right) .
\end{aligned}
$$

Passing to the limit for fixed $\alpha>0$ as $s \rightarrow \infty$ in (4.5) we obtain that

$$
\operatorname{Re}\langle a, z+\alpha b\rangle \geq H_{Q}(a) \text {, i.e., } \operatorname{Re}\langle a, z+\alpha b\rangle=H_{Q}(a) .
$$

Consequently $z+\alpha b \in \partial Q$ for all $\alpha>0$. Since $Q$ is strictly convex at the relative boundary of $\omega$, we have $z+\alpha b \in \omega$ for each $\alpha>0$. Hence the intersection of the supporting hyperplane $\left\{w \in \mathbb{C}^{N} \mid \operatorname{Re}\langle a, w\rangle=H_{Q}(a)\right\}$ to $\bar{Q}$ with $Q$ is unbounded. This contradicts the strict convexity of $Q$ at the relative boundary of $\omega$. Thus there is a neighbourhood $U$ of $a$ in $S$ such that the relation (4.3) holds.

We will show now that there is a neighbourhood of $a$ in $S$ on which $H_{Q}$ is finite. Assume that there are $a_{n} \in S, n \in \mathbb{N}$, such that $\lim _{n \rightarrow \infty} a_{n}=a$ and $H_{Q}\left(a_{n}\right)=\infty$ for all $n \in \mathbb{N}$. Then for each $n \in \mathbb{N}$ there exists $w_{n} \in Q$ with $\operatorname{Re}\left\langle a_{n}, w_{n}\right\rangle>n$. From

$$
\left|w_{n}\right| \geq\left|\left\langle a_{n}, w_{n}\right\rangle\right| \geq\left|\operatorname{Re}\left\langle a_{n}, w_{n}\right\rangle\right|>n \text { for all } n \in \mathbb{N}
$$


it follows that $\lim _{n \rightarrow \infty}\left|w_{n}\right|=\infty$. We select a subsequence $\left(w_{n_{s}}\right)_{s \in \mathbb{N}}$ and $b \in S$ which satisfies $\lim _{s \rightarrow \infty}\left|w_{n_{s}}-z\right|^{-1}\left(w_{n_{s}}-z\right)=b$. For $z_{\alpha, s}$ as above we have

$$
\begin{aligned}
& \operatorname{Re}\left\langle a, z_{\alpha, s}\right\rangle=\left(1-\frac{\alpha}{\left|w_{n_{s}}-z\right|}\right) \operatorname{Re}\langle a, z\rangle+\frac{\alpha}{\left|w_{n_{s}}-z\right|} \operatorname{Re}\left\langle a, w_{n_{s}}\right\rangle \\
& =\left(1-\frac{\alpha}{\left|w_{n_{s}}-z\right|}\right) H_{Q}(a)+\frac{\alpha}{\left|w_{n_{s}}-z\right|} \operatorname{Re}\left\langle a-a_{n_{s}}, w_{n_{s}}\right\rangle \\
& +\frac{\alpha}{\left|w_{n_{s}}-z\right|} \operatorname{Re}\left\langle a_{n_{s}}, w_{n_{s}}\right\rangle \\
& \geq\left(1-\frac{\alpha}{\left|w_{n_{s}}-z\right|}\right) H_{Q}(a)-\frac{\alpha}{\left|w_{n_{s}}-z\right|}\left|a-a_{n_{s}}\right|\left|w_{n_{s}}\right| \\
& +\frac{\alpha n_{s}}{\left|w_{n_{s}}-z\right|} \\
& \geq\left(1-\frac{\alpha}{\left|w_{n_{s}}-z\right|}\right) H_{Q}(a)-\frac{\alpha}{\left|w_{n_{s}}-z\right|}\left|a-a_{n_{s}}\right|\left|w_{n_{s}}\right| .
\end{aligned}
$$

Passing to the limit for fixed $\alpha>0$ as $s \rightarrow \infty$ we obtain that $\operatorname{Re}\langle a, z+$ $\alpha b\rangle \geq H_{Q}(a)$. Since $z+\alpha b \in \bar{Q}$ we conclude that $\operatorname{Re}\langle a, z+\alpha b\rangle=H_{Q}(a)$ and $z+\alpha b \in \omega$ for all $\alpha>0$. This contradicts the strict convexity of $Q$ at the relative boundary of $\omega$. Thus there is a neighbourhood of $a$ in $S$ on which $H_{Q}$ is finite. Since the function $H_{Q}$ is convex and positively homogeneous on $\mathbb{C}^{N}$ there exists an open convex neighbourhood $V$ of $a$ in $\mathbb{C}^{N}$ such that $H_{Q}$ is finite on $V$. By $[\mathbf{1 2}, 2.1 .22] H_{Q}$ is continuous on $V$. Consequently, by (4.3), there is a neighbourhood $W \subset U$ of $a$ in $S$ with

$$
\sup _{w \in(\partial Q) \backslash \omega} \operatorname{Re}\langle w, c\rangle<H_{Q}(c)<\infty \text { for all } c \in W .
$$

The last inequality implies $W \subset S_{\omega}$. This means that $S_{\omega}$ is open in $S$.

(ii): We fix a sequence $\left(w_{n}\right)_{n \in \mathbb{N}} \subset F_{A}$ and select $a_{n} \in A$ with $H_{Q}\left(a_{n}\right)=\operatorname{Re}\left\langle a_{n}, w_{n}\right\rangle$. There are a subsequence $\left(a_{n_{s}}\right)_{s \in \mathbb{N}}$ and $a \in A$ such that $\lim _{s \rightarrow \infty} a_{n_{s}}=a$. We choose $z \in \omega$ with $\operatorname{Re}\langle a, z\rangle=H_{Q}(a)$. Since $H_{Q}<\infty$ on $S_{\omega}$ there is a convex neighbourhood of $a$ in $\mathbb{C}^{N}$ on which the convex function $H_{Q}$ is continuous.

We suppose that $\left(w_{n_{s}}\right)_{s \in \mathbb{N}}$ is unbounded. Without loss of generality $\lim _{s \rightarrow \infty}\left|w_{n_{s}}\right|=\infty$ and the sequence $\left(\left|w_{n_{s}}-z\right|^{-1}\left(w_{n_{s}}-z\right)\right)_{s \in \mathbb{N}}$ converges to a point $b \in S$. For each $\alpha>0$, for large $s \in \mathbb{N}$ the point $z_{\alpha, s}:=$ $z+\alpha\left|w_{n_{s}}-z\right|^{-1}\left(w_{n_{s}}-z\right)$ belongs to $\bar{Q}$ and the following inequalities 
hold:

$$
\begin{aligned}
& \operatorname{Re}\left\langle a, z_{\alpha, s}\right\rangle=\left(1-\frac{\alpha}{\left|w_{n_{s}}-z\right|}\right) \operatorname{Re}\langle a, z\rangle \\
&+\frac{\alpha}{\left|w_{n_{s}}-z\right|} \operatorname{Re}\left\langle a_{n_{s}}, w_{n_{s}}\right\rangle \\
&+\frac{\alpha}{\left|w_{n_{s}}-z\right|} \operatorname{Re}\left\langle a-a_{n_{s}}, w_{n_{s}}\right\rangle \\
& \geq\left(1-\frac{\alpha}{\left|w_{n_{s}}-z\right|}\right) H_{Q}(a)+\frac{\alpha}{\left|w_{n_{s}}-z\right|} \operatorname{Re}\left\langle a_{n_{s}}, w_{n_{s}}\right\rangle \\
&-\frac{\alpha}{\left|w_{n_{s}}-z\right|}\left|a-a_{n_{s}}\right|\left|w_{n_{s}}\right| .
\end{aligned}
$$

Since $\lim _{s \rightarrow \infty} \operatorname{Re}\left\langle a_{n_{s}}, w_{n_{s}}\right\rangle=\lim _{s \rightarrow \infty} H_{Q}\left(a_{n_{s}}\right)=H_{Q}(a)$ and $\lim _{s \rightarrow \infty}\left|a-a_{n_{s}}\right|=0$, passing to the limit for fixed $\alpha>0$ as $s \rightarrow \infty$ in (4.6), we obtain that $\operatorname{Re}\langle a, z+\alpha b\rangle=H_{Q}(a)$ for each $\alpha>0$. As in (i) this contradicts the strict convexity of $Q$ at the relative boundary of $\omega$. Hence the sequence $\left(w_{n_{s}}\right)_{s \in \mathbb{N}}$ is bounded and we can assume that $\left(w_{n_{s}}\right)_{s \in \mathbb{N}}$ converges to $w \in \partial Q$. From the strict convexity of $Q$ at the relative boundary of $\omega$ it follows that $w \in \omega$. Thus $F_{A}$ is compact.

It is easy to see that each $S_{\omega_{n}}$ is closed in $S$. Now the assertion (iii) follows from (ii).

4.5. Theorem. Let $Q \subset \mathbb{C}^{N}$ is convex and locally closed. The following are equivalent:

(i) The algebraic equality $V C\left(\mathbb{C}^{N}\right)=C \bar{V}\left(\mathbb{C}^{N}\right)$ holds.

(i)' The equality $V C\left(\mathbb{C}^{N}\right)=C \bar{V}\left(\mathbb{C}^{N}\right)$ holds algebraically and topologically.

(ii) $Q$ is strictly convex at the relative boundary of $\omega$.

Proof: The conditions (i) and (i)' are equivalent by Theorem 4.1.

(i) $\Rightarrow$ (ii): We assume that $Q$ is not strictly convex at the relative boundary of $\omega$. Then there is $a \in S$ such that the intersection of the support hyperplane $\Pi_{a}:=\left\{z \in \mathbb{C}^{N} \mid \operatorname{Re}\langle a, z\rangle=H_{Q}(a)\right\}$ to $\bar{Q}$ with $Q$ is not compact. Hence there exists an interval $I \subset Q \cap \Pi_{a}$ which is not relatively compact in $Q$. We fix $n \in \mathbb{N}$ with $I \cap Q_{n} \neq \emptyset$. Let $m \geq n$ and $k \in \mathbb{N}$ be arbitrary. There are $w \in I$ with $w \notin Q_{m}$ and $\mu \geq m$ with $w \in Q_{\mu}$. We have

$$
\operatorname{Re}\langle w, a\rangle=H_{Q}(a)=H_{m}(a)=H_{n}(a) .
$$


Fix $b \in S$ and $c \in \mathbb{R}$ such that the hyperplane $\operatorname{Re}\langle z, b\rangle=c$ separates $w$ and $Q_{m}$, i.e.

$$
\operatorname{Re}\langle w, b\rangle>c>H_{m}(b) .
$$

Let $b_{s}:=a+s^{-1} b, a_{s}:=\left|b_{s}\right|^{-1} b_{s}, s \in \mathbb{N}$. For all $s \geq 2$

$$
\begin{aligned}
H_{\mu}\left(a_{s}\right) \geq \operatorname{Re}\left\langle w, a_{s}\right\rangle & =\frac{1}{\left|b_{s}\right|}\left(\operatorname{Re}\langle w, a\rangle+\frac{1}{s} \operatorname{Re}\langle w, b\rangle\right) \\
& >\frac{1}{\left|b_{s}\right|}\left(H_{m}(a)+H_{m}\left(s^{-1} b\right)\right) \\
& =\frac{1}{\left|b_{s}\right|} H_{m}\left(b_{s}\right)=H_{m}\left(a_{s}\right) .
\end{aligned}
$$

Since $\lim _{s \rightarrow \infty} a_{s}=a$ in $\mathbb{C}^{N}$, we have

$$
\lim _{s \rightarrow \infty}\left(H_{m}\left(a_{s}\right)-H_{n}\left(a_{s}\right)\right)=H_{m}(a)-H_{n}(a)=0 .
$$

We fix $p \in \mathbb{N}$ such that

$$
H_{m}\left(a_{p}\right)-H_{n}\left(a_{p}\right)<\frac{1}{2 k} .
$$

Then for all $l \geq 2 k$

$$
H_{m}\left(a_{p}\right)+\frac{1}{l}<H_{n}\left(a_{p}\right)+\frac{1}{2 k}+\frac{1}{l} \leq H_{n}\left(a_{p}\right)+\frac{1}{k} .
$$

Since, by (4.7), $H_{\mu}\left(a_{p}\right)>H_{m}\left(a_{p}\right)$ there is $l \geq 2 k$ with

$$
H_{m}\left(a_{p}\right)+\frac{1}{l}<H_{\mu}\left(a_{p}\right) .
$$

For this $l$, by (4.8) and (4.9), for every $L \in \mathbb{N}$

$$
H_{m}\left(a_{p}\right)+\frac{1}{l}<\min \left(H_{n}\left(a_{p}\right)+\frac{1}{k} ; H_{\mu}\left(a_{p}\right)+\frac{1}{L}\right) .
$$

Consequently the conditions (4.2) and, by Lemma 4.2, (wQ), are not satisfied. By [2, Proposition 2.4 and Theorem 2.7], $V C\left(\mathbb{C}^{N}\right)$ is a proper subset of $C \bar{V}\left(\mathbb{C}^{N}\right)$.

(ii) $\Rightarrow$ (i): Let $Q$ be strictly convex at the relative boundary of $\omega$. If int $Q=\emptyset$ then $Q$ is compact and (4.2) (hence (wQ)) holds. Suppose now that int $Q \neq \emptyset$ and fix $n \in \mathbb{N}$. We will show at first that there is $m \in \mathbb{N}$ such that

$$
H_{m}(a)=H_{Q}(a) \text { for every } a \in S \text { with } H_{m}(a)<H_{n}(a)+\frac{1}{m} .
$$

We suppose the contrary, i.e. for each $m \in \mathbb{N}$ there is $a_{m} \in S$ with

$$
H_{m}\left(a_{m}\right)<H_{n}\left(a_{m}\right)+\frac{1}{m} \text { and } H_{m}\left(a_{m}\right)<H_{Q}\left(a_{m}\right) .
$$


Since $Q_{m}=\operatorname{conv}\left(K_{m} \cup \omega_{m}\right)$ (see Notation 4.3) then $H_{m}=\max \left\{H_{K_{m}} ; H_{\omega_{m}}\right\}$ for all $m \in \mathbb{N}$. Because $H_{K_{m}}>H_{K_{n}}+1 / m$ on $S$ for large $m \in \mathbb{N}$, from (4.10) it follows that

$$
H_{n}\left(a_{m}\right)=H_{\omega_{n}}\left(a_{m}\right) \text { for large } m \in \mathbb{N} .
$$

There exists a subsequence $\left(a_{m_{s}}\right)_{s \in \mathbb{N}}$ which converges to $a \in S$. The equality (4.11) and the continuity of $H_{n}$ imply that $H_{n}(a)=H_{\omega_{n}}(a)$. By (4.10) $a_{m} \notin S_{\omega_{m}}$ for all $m \in \mathbb{N}$.

By Lemma $4.4 S_{\omega}$ is open (in $S$ ) and $\left(S_{\omega_{m}}\right)_{m \in \mathbb{N}}$ is a compact exhaustion of $S_{\omega}$. Hence, if $a \in S_{\omega}$, there is $l \in \mathbb{N}$ such that $a_{m_{s}} \in S_{\omega_{l}}$ for large $s$. This contradicts $a_{m_{s}} \notin S_{\omega_{m}}$ for all $s \in \mathbb{N}$. Consequently $a \notin S_{\omega}$ and $H_{n}(a)<H_{Q}(a)$. Hence there are $w \in Q$ with $\varepsilon:=\operatorname{Re}\langle a, w\rangle-H_{n}(a)>0$ and $s_{0}$ such that

$$
\operatorname{Re}\left\langle a_{m_{s}}, w\right\rangle>H_{n}\left(a_{m_{s}}\right)+\varepsilon / 2 \text { for all } s>s_{0} .
$$

We select $s \in \mathbb{N}$ such that $s>s_{0}, m_{s}>2 / \varepsilon$ and $w \in Q_{m_{s}}$. Then

$$
H_{m_{s}}\left(a_{m_{s}}\right) \geq \operatorname{Re}\left\langle w, a_{m_{s}}\right\rangle>H_{n}\left(a_{m_{s}}\right)+1 / m_{s},
$$

which contradicts (4.10).

Hence there is $m \geq n$ such that the following implication holds:

$$
a \in S, H_{m}(a)<H_{n}(a)+\frac{1}{m} \Rightarrow H_{m}(a)=H_{Q}(a) .
$$

Consequently, condition (4.2) holds with $k:=m$ and $L:=l$ for each $l \in \mathbb{N}$ and $\mu \geq m$. By Lemma 4.2, condition (wQ) is satisfied and, by [2, Proposition 2.4 and Theorem 2.7], $V C\left(\mathbb{C}^{N}\right)$ and $C \bar{V}\left(\mathbb{C}^{N}\right)$ coincide algebraically.

\section{References}

[1] K. D. Bierstedt, A survey of some results and open problems in weighted inductive limits and projective description for spaces of holomorphic functions, Hommage à Pascal Laubin, Bull. Soc. Roy. Sci. Liège 70(4-6) (2001), 167-182 (2002).

[2] K. D. Bierstedt and J. Bonet, Weighted (LF)-spaces of continuous functions, Math. Nachr. 165 (1994), 25-48.

[3] K. D. Bierstedt, R. Meise and W. H. Summers, A projective description of weighted inductive limits, Trans. Amer. Math. Soc. 272(1) (1982), 107-160.

[4] J. Bonet and R. Meise, Ultradistributions of Roumieu type and projective descriptions, J. Math. Anal. Appl. 255(1) (2001), $122-136$. 
[5] J. Bonet And R. Meise, Quasianalytic functionals and projective descriptions, Math. Scand. 94(2) (2004), 249-266.

[6] J. Bonet, R. Meise and S. N. Melikhov, Holomorphic functions on locally closed convex sets and projective descriptions, Bull. Belg. Math. Soc. Simon Stevin 10(4) (2003), 491-503.

[7] J. Bonet, R. Meise And S. N. Melikhov, Projective representations of spaces of quasianalytic functionals, Studia Math. 164(1) (2004), 91-102.

[8] H. Cartan, Variétés analytiques réelles et variétés analytiques complexes, Bull. Soc. Math. France 85 (1957), 77-99.

[9] L. EHrEnPREIs, Solution of some problems of division. IV. Invertible and elliptic operators, Amer. J. Math. 82 (1960), 522-588.

[10] L. EHRENPREIS, "Fourier analysis in several complex variables", Pure and Applied Mathematics XVII, Wiley-Interscience Publishers A Division of John Wiley \& Sons, New York-London-Sydney 1970.

[11] L. Hörmander, On the range of convolution operators, Ann. of Math. (2) 76 (1962), 148-170.

[12] L. Hörmander, "Notions of convexity", Progress in Mathematics 127, Birkhäuser Boston, Inc., Boston, MA, 1994.

[13] Yu. F. KorobeINIK, Solvability of the convolution equation in some classes of analytic functions, (Russian), Mat. Zametki 49(2) (1991), 74-83, 159; translation in: Math. Notes 49(1-2) (1991), $165-172$.

[14] I. M. MAL'TSEV, Epimorphicity of a convolution operator in spaces of analytic functions on connected sets, (Russian), Dokl. Akad. Nauk 336(3) (1994), 297-300; translation in: Russian Acad. Sci. Dokl. Math. 49(3) (1994), 501-506.

[15] A. Martineau, Sur la topologie des espaces de fonctions holomorphes, Math. Ann. 163 (1966), 62-88.

[16] A. Martineau, Équations différentielles d'ordre infini, Bull. Soc. Math. France 95 (1967), 37-47.

[17] R. MEISE AND D. VOGT, "Introduction to functional analysis", Translated from the German by M. S. Ramanujan and revised by the authors, Oxford Graduate Texts in Mathematics 2, The Clarendon Press, Oxford University Press, New York, 1997.

[18] S. N. Melikhov and S. Momm, Analytic solutions of convolution equations on convex sets with an obstacle in the boundary, Math. Scand. 86(2) (2000), 293-319.

[19] V. V. Napalkov and I. A. Rudakov, A convolution operator in the space of real analytic functions, (Russian), Mat. Zametki 49(3) 
(1991), 57-65, 159; translation in: Math. Notes 49(3-4) (1991), 266-271.

[20] L. I. Ronkin, "Introduction to the theory of entire functions of several variables", Translated from the Russian by Israel Program for Scientific Translations, Translations of Mathematical Monographs 44, American Mathematical Society, Providence, R.I., 1974.

[21] D. Vogt, Regularity properties of (LF)-spaces, in: "Progress in functional analysis" (Peñíscola, 1990), North-Holland Math. Stud. 170, North-Holland, Amsterdam, 1992, pp. 57-84.

José Bonet:

Departamento de Matemática Aplicada and Instituto de Matemática Pura y Aplicada

Universidad Politécnica de Valencia

E-46071, Valencia

Spain

E-mail address: jbonet@mat.upv.es

Reinhold Meise:

Mathematisches Institut

Heinrich-Heine-Universität

Universitätsstraße 1

D-40225 Düsseldorf

Germany

E-mail address: meise@math.uni-duesseldorf.de

Sergej N. Melikhov:

Department of Mechanics and Mathematics

Rostov State University

Zorge st. 5

344090 Rostov on Don

Russia

E-mail address: melih@ms.math.rsu.ru

Rebut el 23 de febrer de 2005. 